\title{
Carrier transfer between confined and localized states in type II InAs/GaAsSb quantum wells
}

\author{
M. Dyksik' ${ }^{1}$ M. Motyka1 $~$ R. Weih ${ }^{2}$ S. Höfling ${ }^{2,3}$ • \\ M. Kamp ${ }^{2} \cdot$ G. Sęk ${ }^{1} \cdot$ J. Misiewicz ${ }^{1}$
}

Received: 22 April 2016/Accepted: 5 January 2017/Published online: 16 January 2017

(C) The Author(s) 2017. This article is published with open access at Springerlink.com

\begin{abstract}
Temperature-resolved photoluminescence studies were performed on tenselystrained AlSb/InAs/GaAsSb W-shaped type II quantum wells. They revealed two emission bands: one at lower energy of localized origin resulting from carrier trapping states at interfaces and dominates at low-temperature; and one corresponding to the fundamental optical transition in the type II quantum well. With the temperature increase to $170-200 \mathrm{~K}$ the low-energy emission is quenched and the high-energy band dominates and its intensity increases, indicating carrier transfer processes between the respective states at elevated temperatures. In addition, the integrated photoluminescence intensity was measured as a function of excitation power. At high excitation regime the emission intensity of the lowenergy emission band saturated, indicating low density of states, thus confirming its localized nature. The depth of the localization potential at the InAs/GaAsSb interface was determined to be $13-15 \mathrm{meV}$.
\end{abstract}

Keywords Fourier-transform infrared spectroscopy - Type II quantum wells · Photoluminescence quenching $\cdot$ Carrier transfer $\cdot$ Localized states

This article is part of the Topical Collection on TERA-MIR: Materials, Generation, Detection and Applications.

Guest Edited by Mauro F. Pereira, Anna Wojcik-Jedlinska, Renata Butkute, Trevor Benson, Marian Marciniak and Filip Todorov.

M. Dyksik

mateusz.dyksik@pwr.edu.pl

1 Laboratory for Optical Spectroscopy of Nanostructures, Division of Experimental Physics, Faculty of Fundamental Problems of Technology, Wrocław University of Science and Technology, Wybrzeże Wyspiańskiego 27, Wrocław 50-370, Poland

2 Technische Physik, Wilhelm-Conrad-Röntgen-Research Center for Complex Material Systems, University of Würzburg, Am Hubland, Würzburg 97074, Germany

3 SUPA, School of Physics and Astronomy, University of St. Andrews, North Haugh, St. Andrews KY16 9SS, UK 


\section{Introduction}

Interband cascade lasers (ICL) with an active region consisting of type II quantum wells emerged as attractive laser sources for applications in sensors of natural and industrial gases, which have absorption lines in the mid-infrared. As a result, sensors with ICLs and operating on the basis of the tunable diode laser absorption spectroscopy are of great demand. To date, several types of such devices have been demonstrated, including sensors of formaldehyde (Lundqvist et al. 2012), nitric oxide (Von Edlinger et al. 2014) and methane (Dong et al. 2016). The standard approach to design the active region is to take advantage of the so-called "W-shaped" alignment. In order to realize it, a broken-gap InAs/GaInSb/InAs system is employed, with InAs and GaInSb layers for the confinement of electrons and holes, respectively (Meyer et al. 1995; Yang 1995). As an electron's barrier an AlSb layer is used, resulting in the AlSb/InAs/GaInSb/InAs/AlSb material combination. The thickness of individual layers is usually of single nanometers and extraordinary accuracy is required, since, for instance, the InAs thickness variation of 1 monolayer usually shifts the emission wavelength by hundreds of nanometers (Dyksik et al. 2015).

Recently, the idea of a polarization independent mid-infrared ICL has been presented (Motyka et al. 2016; Ryczko et al. 2015). In such a device, emission intensities of transverse electric and transverse magnetic polarizations are equal, resulting from the proper selection of heavy and light hole contributions to the ground state transition. Realization of such a scheme requires a new design of layers in order to enhance the light hole admixture. A compressively strained GaInSb layer for hole confinement is replaced with a tensely strained GaAsSb layer, resulting in pushing the light hole states towards the valence band edge of GaAsSb.

In this work, the optical properties of tensely-strained AlSb/InAs/GaAsSb type II quantum wells are investigated. The temperature-resolved photoluminescence measurements were performed in order to study the transfer of carriers within the quantum wells. The analysis of the obtained data indicates two emission bands. Throughout this report the origin of both emission bands is discussed. At low temperatures, only the low-energy one is visible. When the lattice temperature is increased, the high-energy band increases in intensity and finally dominates, indicating on the possible carrier transfer processes between the two kinds of states. The origin of the emission bands has additionally been confirmed by the excitation power dependence.

\section{Materials and methods}

The investigated samples were designed to mimic the active region of an interband cascade laser. In an operational device such active region is placed in between injector stages for both electrons and holes and sandwiched by cladding layers. As a result a full ICL is a complex multilayered structure. In order to simplify the process of design verification, structures imitating the active region were grown and studied before the fabrication of a final ICL. The investigated samples were grown on a (100)-oriented GaSb substrate, in a solid source molecular beam epitaxy system equipped with valved cracker cells for both antimony and arsenic. On the substrate the so called "W-like" shape quantum wells were grown, consisting of two InAs layers and one GaAsSb layer. Two samples A and B were studied with the same $2 \mathrm{~nm}$ InAs layer thickness in both samples. The thickness of the 
GaAsSb layer was 3 and $8 \mathrm{~nm}$ for the samples $\mathrm{A}$ and $\mathrm{B}$, and the molar content of arsenic was set to 5 and $8 \%$, respectively. The active region is surrounded by $2.5 \mathrm{~nm}$ thick AlSb barriers. In order to enhance the overall optical response, the wells were repeated five times and separated from each other by $20 \mathrm{~nm}$ of GaSb. The entire structure is terminated by the $\mathrm{GaSb}$ layer of the same thickness. For the band alignment and energy structure calculations the reader is referred to (Motyka et al. 2016; Ryczko et al. 2015). As a reference sample, the AlSb/InAs/Ga $\mathrm{Ga}_{0.7} \mathrm{In}_{0.3} \mathrm{Sb} / \mathrm{InAs} / \mathrm{AlSb}$ "W-like" shape quantum well has been taken with the InAs and GaInSb thickness of 1.4 and $3.5 \mathrm{~nm}$, respectively. All the structural data (molar content of arsenic, layers thickness) of the analyzed QWs are based on the highresolution X-ray diffraction analysis and the growth calibration procedures.

The optical studies were performed with a Bruker Fourier-transform infrared spectrometer Vertex 80v operating in the step-scan mode. A liquid nitrogen cooled InSb photodetector was used for the PL measurements. An external pump beam provided by a $660 \mathrm{~nm}$ semiconductor laser diode was mechanically chopped at a frequency of $275 \mathrm{~Hz}$. This allows for a phase sensitive detection of the optical response using a lock-in amplifier. The temperature resolved photoluminescence studies were performed with a continuous flow liquid helium cryostat with $\mathrm{CaF}_{2}$ windows. For more information about the measurement setup the reader is referred to previous works (Motyka et al. 2011, 2009; Motyka and Misiewicz 2010).

\section{Results and discussion}

Figure 1 presents normalized PL spectra measured at the temperature of $170 \mathrm{~K}$ where two bands of interest are visible. The QW ground state emission of the samples A and B is located at about 0.47 and $0.5 \mathrm{eV}$. According to previous considerations, the energy of the

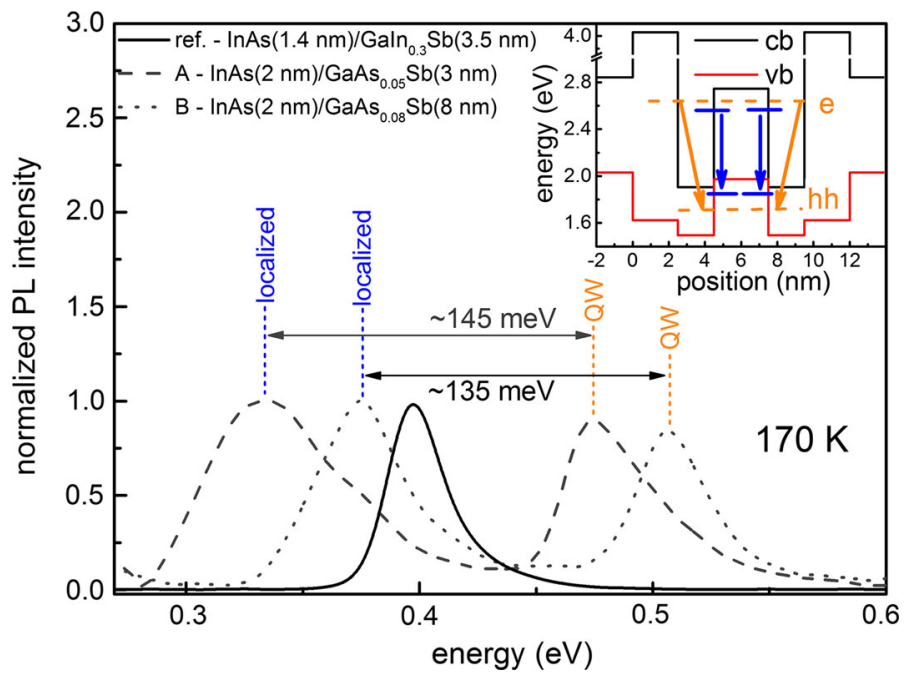

Fig. 1 Photoluminescence spectra for the reference InAs/GaInSb sample (solid) and two samples with GaAsSb layer of different thickness and As content (see legend). (inset) Band alignment: $c b$ and $v b$ stand for conduction band edge and valence band edge, respectively. Orange and blue arrows indicate the QW and localized transitions 
fundamental transition is very sensitive to the thickness of the InAs layer, while the thickness of the layer for hole confinement (GaAsSb or GaInSb) is of minor importance in that respect. Since the thickness of the InAs layers is constant, the widening of the layer for holes confinement from 3 to $8 \mathrm{~nm}$ should result in a red-shirt of fundamental transition. Even so, in Fig. 1 one can notice a blue-shirt of QW transition. Such behaviour of the ground transition is induced by the content of arsenic equal to 5-8\% for samples $\mathrm{A}$ and $\mathrm{B}$, influencing both the strain and band gap of GaAsSb and thus the InAs/GaAsSb valence band offset. Both spectra for the samples A and B exhibit a low-energy emission band at about 0.33 and $0.38 \mathrm{eV}$ at $170 \mathrm{~K}$, respectively. In the case of both samples, the low-energy band lays 145 and $135 \mathrm{meV}$ below the ground state emission, respectively. This lowenergy emission band is not seen in the reference type II QW structure with the GaInSb layer for the confinement of holes (gray dashed), thus its origin is assigned to some intrinsic properties of the GaAsSb layer. Inset to Fig. 1 shows the band alignment of type II QW with (in orange) the position of the initial (e) and final (hh) states of QW transition. The plausible location of the low-energy transition is marked by blue arrows.

The evolution of PL spectra was measured as a function of temperature. Data obtained for the sample $\mathrm{A}$ are presented in Fig. $2 \mathrm{~b}$ in a form of a map. Two emission bands are

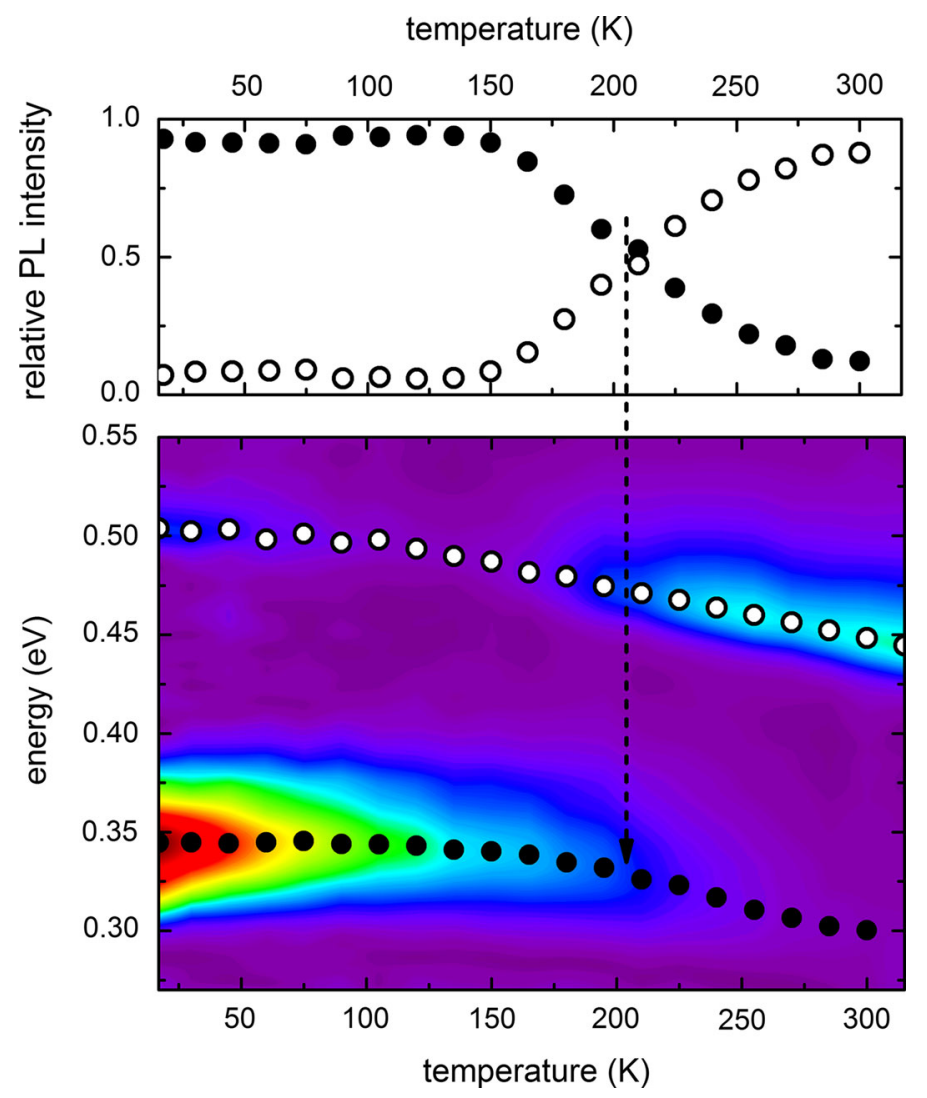

Fig. 2 The contribution of emission from localized (solid) and confined (open) states to the total photoluminescence signal (top). The temperature resolved photoluminescence map for the sample A (bottom) 
visible, with the one at $0.35 \mathrm{eV}$ dominating at low temperature of $15 \mathrm{~K}$. A similar band has been visible in the emission spectra from type II QWs with quaternary GaInAsSb layer (Janiak et al. 2013). Its origin was assigned to localization of carriers at the InAs/GaInAsSb interface due to the intermixing of arsenic atoms (Motyka et al. 2015). Also earlier considerations regarding InAs/GaAsSb heterojunctions (Moiseev et al. 2010) indicated the low-energy emission might be connected with localization of carriers at the InAs/GaAsSb interface. The emission from the QW emerges at elevated temperature, around $200 \mathrm{~K}$, at about $0.47 \mathrm{eV}$ and is noticeable up to the ambient temperature. Although both emission bands are separated by $145 \mathrm{meV}$, Fig. 2a presents a clear indication on the carrier transfer between the localized and quantum well states. The relative PL intensity of the localized states (LS), understood as the contribution to the total emission, is constant with the temperature up to $165 \mathrm{~K}$, with a moderate trend towards lower values beyond that. While the LS emission preliminary quenches at $165 \mathrm{~K}$, the QW emission is thermally activated. The contribution to the total emission resulting from the QW is enhanced and sub-linearly increases up to the ambient temperature. At $210 \mathrm{~K}$ the QW and LS emissions have similar intensities. In the case of the sample B the two bands cross at $170 \mathrm{~K}$.

Figure 3 shows the Arrhenius plot for the LS and QW emissions in solid and open circles, respectively (sample A). The experimental data regarding the LS emission is fitted with the following well-known formula:

$$
I(T)=\frac{I(0)}{1+\sum_{i=1}^{n} C_{i} \exp \frac{-E_{i}}{k T}}
$$

where, in denominator, $n$ stands for the number of thermally activated processes, with activation energies $E_{i}$ and amplitude parameters $C_{i}$. The fitting procedure was performed assuming two independent activation processes reflected by two energies $E_{L S(1)}$ and $E_{L S(2)}$. The former energy equals to $155.1 \mathrm{meV}$, corresponding to the energy distance between two emission bands of interest in Fig. 1 and reflecting the process of carriers leaving the trap states in favor of the confined states within quantum well. The second activation energy of

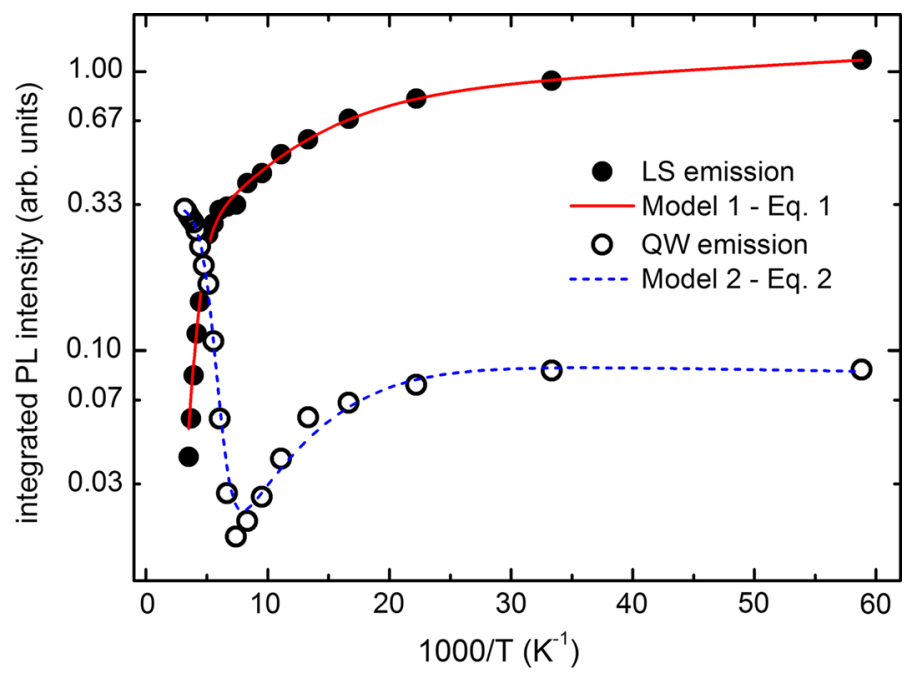

Fig. 3 Integrated PL intensity (log scale) as a function of the inverse temperature for the $\operatorname{InAs} / \mathrm{GaAs}_{0.05} \mathrm{Sb}$ (sample A) type II QW 
$14.3 \mathrm{meV}$ is attributed with the depth of localization potential at the InAs/GaAsSb interface. When the sample temperature reaches the $165 \mathrm{~K}$, which translates into $14.3 \mathrm{meV}$ in the thermal energy scale, carriers tend to escape from the LS potential to the QW ground state. Hence the localization energy is derived to be $14.3 \mathrm{meV}$. As previously mentioned, the QW emission (open circles) exhibits different behavior. In order to perform fitting procedure, a following model with two activation energies is employed:

$$
I(T)=\frac{I_{1}(0)}{1+C_{1} \exp \frac{-E_{Q W(1)}}{k T}}+\frac{I_{2}(0)}{1+C_{2} \exp \frac{-E_{Q W(2)}}{k T}}
$$

The first term describes the high-temperature region in Fig. 2 where the QW emission dominates. The activation energies extracted from the fitting procedure equal to -147 and $28 \mathrm{meV}$. The absolute value of the former one, as previously, corresponds to the energy distance between both emission bands in the PL spectrum (see Fig. 1). The negative value reflects the direction of the carrier transfer from the localization potential towards the quantum well, i.e. an increase in the QW emission intensity with temperature. The second activation energy affecting the QW emission at high temperatures corresponds to the tunneling of thermally activated holes from the confined state in the GaAsSb layer to the valence band of the GaSb spacing layer. According to previous investigations (Motyka et al. 2016), in case of structures with GaAsSb layers, the ground hole state is located in the vicinity of the valence band edge of GaSb spacing layer (approx. 30-50 meV). Similar process involving heavy holes has been already studied for type II InAs/GaInSb QWs based on GaSb (Sęk et al. 2011) and InAs (Dyksik et al. 2016) substrates. In the case of the sample B, similar consideration was performed. Now, the energy corresponding to the depth of the localization potential, $E_{L S(1)}$, equals to $13 \mathrm{meV}$, corresponding to the lattice temperature of $150 \mathrm{~K}$ at which carriers tend to escape from the trap states to the QW. The energy $E_{L S(2)}$ yet again is understood as the energy distance between the two emission bands and equals to $135 \mathrm{meV}$. The further two energies, $E_{Q W(1)}$ and $E_{Q W(2)}$, describing the behavior of QW emission according to Eq. 2, equal to -137 and $26 \mathrm{meV}$, respectively. As in the case of the sample A, the former one corresponds to the energy distance between the QW and LS emission bands, whereas the latter one describes the process of holes tunneling from the heavy hole state into the valence band of GaSb spacer and has been already studied elsewhere (Sęk et al. 2011).

Further investigations were performed in order to prove the localized nature of the lowenergy emission. Figure 4a presents the integrated PL intensity as a function of the excitation power for the sample A. The dependence for the localized state measured at low temperature of $12 \mathrm{~K}$ is compared with the QW emission at $200 \mathrm{~K}$. The QW line intensity changes linearly with the excitation, while the former one depends sub-linearly, i.e. has a saturation character in linear scale. Since the density of states in the localization potential is orders of magnitude lower compared to the QW's confined states, the trap states are filled with much lower rate than the QW ones. This is an additional argument for the concluded origin of the low energy peaks appearing after addition of arsenic, the emission intensity is expected to saturate at significantly lower generation rates (excitation power densities). For reference, the emission from the GaSb substrate is presented at both temperatures of 12 and $200 \mathrm{~K}$ and exhibits similar behavior to the QW one. The reduced density of states of the localization potential is directly presented in Fig. 4b. As the excitation energy increases the trapping potential is filled with carriers and the emission from the localized state blue-shifts, whilst the QW one remains constant. The shift of the LS peak energy provides information about the depth of localization potential of $16 \mathrm{meV}$, 


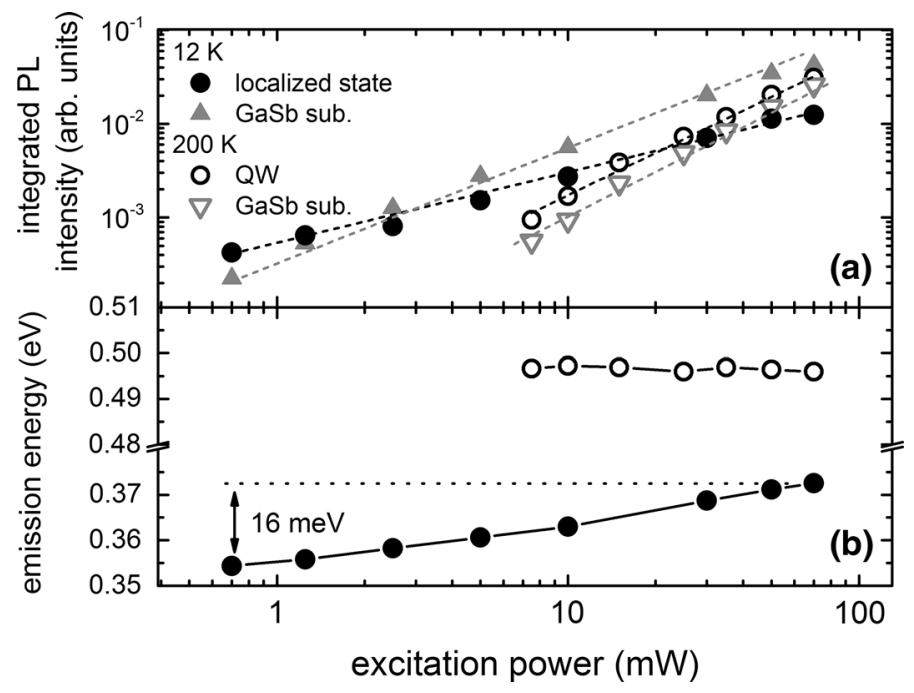

Fig. 4 Photoluminescence peak integral intensity $\mathbf{a}$ and transition energy $\mathbf{b}$ as a function of the excitation power. Note the log-log and log scales for upper and lower panels, respectively. Dashed lines are guides to the eye

being in good agreement with temperature resolved studies. In the case of the sample B analogous studies were performed. The excitation power dependence of the integrated PL intensities was found to follow the same tendency as for sample A.

\section{Conclusions}

We have performed the characterization of the emission properties of AlSb/InAs/GaAsSb W-shaped type II quantum wells with different thickness and As content of a GaAsSb layer in the spectral region of mid-infrared. The photoluminescence studies revealed two emission bands at low- and high-energy side of the spectrum, connected with localized and confined states, respectively. At low temperatures only emission from the low-energy band was observed assigned as localized emission The localization potential was found to be $13-15 \mathrm{meV}$. At the lattice temperature of $150-170 \mathrm{~K}$ carriers are gradually released and transferred to the well, hence the emission band related to the confined states within the quantum well appears and increases with temperature.

Acknowledgements The work has been supported from iCspec project, which received funding from the European Commission's Horizon 2020 Research and Innovation Programme under grant agreement No. 636930, and also by the National Science Centre of Poland within Grant No. 2014/15/B/ST7/04663.

Open Access This article is distributed under the terms of the Creative Commons Attribution 4.0 International License (http://creativecommons.org/licenses/by/4.0/), which permits unrestricted use, distribution, and reproduction in any medium, provided you give appropriate credit to the original author(s) and the source, provide a link to the Creative Commons license, and indicate if changes were made. 


\section{References}

Dong, L., Li, C., Sanchez, N.P., Gluszek, A.K., Griffin, R.J., Tittel, F.K.: Compact CH4 sensor system based on a continuous-wave, low power consumption, room temperature interband cascade laser. Appl. Phys. Lett. 108, 11106 (2016)

Dyksik, M., Motyka, M., Kurka, M., Ryczko, K., Dallner, M., Höfling, S., Kamp, M., Sęk, G., Misiewicz, J.: Photoluminescence quenching mechanisms in type II InAs/GaInSb QWs on InAs substrates. Opt. Quantum Electron. 48, 401 (2016)

Dyksik, M., Motyka, M., Sęk, G., Misiewicz, J., Dallner, M., Weih, R., Kamp, M., Höfling, S.: Submonolayer uniformity of type II InAs/GaInSb W-shaped quantum wells probed by full-wafer photoluminescence mapping in the mid-infrared spectral range. Nanoscale Res. Lett. 10, 402 (2015)

Von Edlinger, M., Scheuermann, J., Weih, R., Zimmermann, C., Nähle, L., Fischer, M., Koeth, J., Höfling, S., Kamp, M.: Monomode interband cascade lasers at $5.2 \mu \mathrm{m}$ for nitric oxide sensing. IEEE Photonics Technol. Lett. 26, 480-482 (2014)

Janiak, F., Motyka, M., Sęk, G., Dyksik, M., Ryczko, K., Misiewicz, J., Weih, R., Höfling, S., Kamp, M., Patriarche, G.: Effect of arsenic on the optical properties of GaSb-based type II quantum wells with quaternary GaInAsSb layers. J. Appl. Phys. 114, 223510 (2013)

Lundqvist, S., Kluczynski, P., Weih, R., von Edlinger, M., Nähle, L., Fischer, M., Bauer, A., Höfling, S., Koeth, J.: Sensing of formaldehyde using a distributed feedback interband cascade laser emitting around 3493 nm. Appl. Opt. 51, 6009-6013 (2012)

Meyer, J.R., Hoffman, C.A., Bartoli, F.J., Ram-Mohan, L.R.: Type-II quantum-well lasers for the midwavelength infrared. Appl. Phys. Lett. 67, 757 (1995)

Moiseev, K., Ivanov, E., Romanov, V., Mikhailova, M., Yakovlev, Y., Hulicius, E., Hospodková, A., Pangrác, J., Šimeček, T.: Intense interface luminescence in type II narrow-gap InAs-based heterostructures at room temperature. Phys. Procedia. 3, 1189-1193 (2010)

Motyka, M., Dyksik, M., Ryczko, K., Weih, R., Dallner, M., Höfling, S., Kamp, M., Sęk, G., Misiewicz, J.: Type-II quantum wells with tensile-strained GaAsSb layers for interband cascade lasers with tailored valence band mixing. Appl. Phys. Lett. 108, 101905 (2016)

Motyka, M., Misiewicz, J.: Fast differential reflectance spectroscopy of semiconductor structures for infrared applications by using Fourier transform spectrometer. Appl. Phys. Express. 3, 112401 (2010)

Motyka, M., Sęk, G., Janiak, F., Misiewicz, J., Kłos, K., Piotrowski, J.: Fourier-transformed photoreflectance and fast differential reflectance of $\mathrm{HgCdTe}$ layers. The issues of spectral resolution and Fabry-Perot oscillations. Meas. Sci. Technol. 22, 125601 (2011)

Motyka, M., Sęk, G., Misiewicz, J., Bauer, A., Dallner, M., Höfling, S., Forchel, A.: Fourier Transformed photoreflectance and photoluminescence of mid infrared GaSb-Based Type II quantum wells. Appl. Phys. Express. 2, 126505 (2009)

Motyka, M., Sęk, G., Ryczko, K., Dyksik, M., Weih, R., Patriarche, G., Misiewicz, J., Kamp, M., Höfling, S.: Interface intermixing in type II InAs/GaInAsSb quantum wells designed for active regions of midinfrared-emitting interband cascade lasers. Nanoscale Res. Lett. 10, 471 (2015)

Ryczko, K., Sęk, G., Misiewicz, J.: Novel design of type-II quantum wells for mid-infrared emission with tensile-strained GaAsSb layer for confinement of holes. Appl. Phys. Express. 8, 121201 (2015)

Sęk, G., Janiak, F., Motyka, M., Ryczko, K., Misiewicz, J., Bauer, A., Höfling, S., Forchel, A.: Carrier loss mechanisms in type II quantum wells for the active region of GaSb-based mid-infrared interband cascade lasers. Opt. Mater. (Amst) 33, 1817-1819 (2011)

Yang, R.Q.: Infrared laser based on intersubband transitions in quantum wells. Superlattices Microstruct. 17, 77-83 (1995) 